\title{
SINDROME DE BURNOUT E SUAS CONSEQUENCIAS NOS PROFISSIONAIS DE ENFERMAGEM
}

\section{BURNOUT SYNDROME AND ITS CONSEQUENCES IN PROFESSIONAL NURSING}

\author{
Clecilene Gomes CARVALHO ${ }^{1}$ \\ Sérgio Ricardo MAGALHÃES ${ }^{2}$
}

\begin{abstract}
RESUMO: Burnout é uma palavra inglesa que se refere a algo que deixou de funcionar por exaustão. É um problema que atinge profissionais de serviço, principalmente aqueles voltados para atividades de cuidado com outros, em que a oferta do cuidado ou serviço freqüentemente ocorre em situações de mudanças emocionais. A Síndrome de Burnout assume uma concepção multidimensional, cuja manifestação se caracteriza por esgotamento emocional, redução da realização pessoal no trabalho e despersonalização do outro. Levando-se em consideração que enfermeiros, técnicos e auxiliares de enfermagem, constituem um grupo com grande predisposição ao desenvolvimento da Síndrome, por serem os profissionais da saúde que mais tempo passam em contato com o paciente e com seus familiares dentro do ambiente de trabalho em situações de constantes mudanças emocionais, foi realizado um estudo de revisão bibliográfica. A finalidade deste estudo foi a de levantar informações sobre os principais fatores de risco que favorecem o aparecimento da Síndrome de Burnout e sua conseqüência para o indivíduo, organização e sociedade. Este trabalho servirá de subsidio para reflexões e debates, tanto para os profissionais envolvidos, quanto para os gestores e futuros profissionais da área.
\end{abstract}

Palavras chave: Síndrome de Burnout. Saúde do Trabalhador. Esgotamento profissional. Profissional de enfermagem.

\begin{abstract}
Burnout is an English word that refers to something that crashed from exhaustion. It is a problem that affects service professionals, particularly those facing care activities with others, in which the provision of care or service often occurs in situations of emotional changes. The syndrome takes a multidimensional concept, whose manifestation is characterized by emotional exhaustion, reduced personal accomplishment and depersonalization of the other. Taking into consideration that nurses, technicians and nursing assistants, are a great group with a predisposition to develop the syndrome because they are health professionals who spend more time in contact with the patient and their family members within the work environment emotional situations of constant change, we performed a bibliographic review in order to gather information on key risk factors that favor the onset of the Burnout syndrome and its consequences for the individual, organization and society. This work will serve as a subsidy for reflection and discussion, both the professionals involved, as future managers and professionals.
\end{abstract}

Keywords: Burnout Syndrome. Occupational Health. Burnout. Nurse.

\footnotetext{
${ }^{1}$ Graduada em Enfermagem pela Universidade Vale do Rio Verde (Unincor), campus Betim. Membro da Associação Mineira de Hipertensão Pulmonar - AMIHAP. E-mail: clecilene@ globo.com.

2 Doutor em Engenharia Biomédica, docente do curso Enfermagem da Universidade Vale do Rio Verde (UninCor), campus Betim. E-mail: serrgio.magalhaes@unincor.edu.br 


\section{INTRODUÇÃO}

Novas configurações organizacionais têm demandado, em diferentes graus e por entre os diversos setores produtivos, novas exigências de qualidade na execução das tarefas, mais qualificação e novas competências do trabalhador. Tais demandas incidem particularmente no setor de serviços, face às suas peculiaridades, como o caráter direto do relacionamento do trabalhador com o cliente ou usuário e a diversidade das informações. Essas demandas são ainda maiores nos serviços de saúde em decorrência da ênfase na nobreza da missão dessas organizações e da busca por equacionar preceitos éticos e racionalidade técnica. Além disso, os serviços de atenção à saúde guardam especificidades relativas ao trato com a dor, ao sofrimento e ao mal-estar orgânico, emocional e social das pessoas. Portanto, requer dos profissionais uma carga adicional de competências interpessoais, além das condições inerentes ao exercício profissional que incluem trabalho em turnos e escalas com fortes pressões externas. Dessa maneira, novas enfermidades como a Síndrome de Burnout

(SB), surgem decorrentes das mudanças introduzidas no mundo do trabalho (BORGES et al, 2006).

O trabalho é uma atividade que pode ocupar grande parcela do tempo de cada indivíduo e do seu convívio em sociedade.
Dejours (1992), afirmava que o trabalho nem sempre possibilita realização profissional. Pode, ao contrário, causar problemas desde insatisfação até exaustão (TRIGO et $a l$, 2007).

O termo de origem inglesa Burnout, designa algo que deixou de funcionar por exaustão de energia. Pode-se dizer que o termo descreve uma síndrome com características associadas aos fatores de exaustão e esgotamento, que representam uma resposta aos estressores laborais crônicos (SILVEIRA et al, 2005).

A SB assume uma concepção multidimensional, cuja manifestação se caracteriza por esgotamento emocional, redução da realização pessoal no trabalho e despersonalização do outro (BORGES et al, 2002).

Levando-se em consideração que enfermeiros, técnicos e auxiliares de enfermagem, constituem um grupo com grande predisposição ao desenvolvimento da $\mathrm{SB}$, por serem os profissionais da saúde que mais tempo passam em contato com o paciente e com seus familiares dentro do ambiente de trabalho e em situações de constantes mudanças emocionais, foi realizado um estudo de revisão bibliográfica. A finalidade deste estudo foi a de levantar informações sobre os principais fatores de risco que favorecem o aparecimento da SB e sua conseqüência para o indivíduo, a 
organização e a sociedade.

Este trabalho servirá de subsidio para reflexões e debates, tanto dos profissionais envolvidos, quanto gestores e futuros profissionais da área.

\section{METODOLOGIA}

Realizou-se revisão bibliográfica utilizando-se a base de dados da La Literatura Latino Americana e do Caribe em Ciências da Saúde (Lilacs), Scientific Electronic Library Online (Scielo), National Library of Medicine (Medline), do Centro Latino Americano e do Caribe de Informação em Ciências da Saúde (BIREME). Utilizaram-se os unitermos: Burnout, nursing, síndrome, fatores de risco, consequiência, indivíduo, esgotamento profissional, organização, sociedade, epidemiologia, enfermagem, saúde do trabalhador. A busca foi feita para o período compreendido entre 2002 a 2011, cruzando-se o unitermo Burnout com os outros citados e selecionando-se artigos publicados em língua portuguesa e inglesa.

Após a seleção dos artigos, fez-se busca ativa entre as citações bibliográficas para identificar artigos de relevância que não tivessem aparecido no primeiro levantamento. Selecionaram-se artigos epidemiológicos, conceituais e de revisão que relacionassem o Burnout, seus aspectos conceituais e comorbidades aos trabalhadores da área de enfermagem. Foram excluídos artigos que abordaram outras categorias profissionais com exceção às que preencheram aos critérios citados.

\section{RESULTADOS E DISCUSSÃO}

Para Borges et al (2002), a SB é um processo que se desenvolve na interação de características do ambiente de trabalho e características pessoais. É um problema que atinge profissionais de serviço, principalmente aqueles voltados para atividades de cuidado com outros, no qual a oferta do cuidado ou serviço freqüentemente ocorre em situações de mudanças emocionais. Ajudar outras pessoas traz custos emocionais que, só recentemente, tem sido dada atenção para estes na realização do objetivo. O exercício destas profissões implica uma relação com o cliente permeada de ambigüidades, como conviver com a tênue distinção entre envolver-se profissional e não pessoalmente na ajuda ao outro.

É importante salientar que a palavra estresse não pode ser confundida com Burnout no que se refere aos conceitos e diferenças, pois estresse ocorre a partir de reações do organismo às agressões de origens diversas, capazes de perturbar o equilíbrio interno do ser humano. Em contrapartida, Burnout é a resposta do estresse laboral crônico que envolve atitudes e alterações 
comportamentais negativas relacionadas ao contexto de trabalho com desconsideração do lado humano. No caso de trabalhadores de enfermagem, atinge os pacientes, organização e o próprio trabalho, isto acontece quando os métodos de enfrentamento contra os fatores estressantes falham ou são insuficientes (JONAS; HADDAD, 2009).

\section{O Burnout não aparece} repentinamente como resposta a um estressor determinado, mas emerge de uma seqüência determinada de fatores desencadeantes. $\mathrm{O}$ sofrimento surge assim que a relação do homem com a organização do trabalho é permanentemente bloqueada (NUNES, 2008).

Com Burnout o indivíduo perde a capacidade de compreender o sentimento ou reação da outra pessoa e ainda a faculdade de compreender emocionalmente o outro. Não se deixa envolver com os problemas e as dificuldades dos outros e as relações interpessoais são cortadas, como se ele estivesse em contato apenas com objetos, ou seja, a relação torna-se desprovida de calor humano, não tem empatia.

$$
\text { Trata-se de uma síndrome }
$$
multidimensional, caracterizada por três componentes: exaustão emocional, diminuição da realização pessoal e despersonalização. $\mathrm{O}$ primeiro refere-se a sentimentos de fadiga e redução dos recursos emocionais necessários para lidar com a situação estressora. O segundo refere-se à percepção de deterioração da auto competência e falta de satisfação com as realizações e os sucessos de si próprio no trabalho. O terceiro componente refere-se a atitudes negativas, ceticismo, insensibilidade e despreocupação com respeito a outras pessoas. Todos os fatores são preocupantes e suscetíveis a causar danos, tanto nos indivíduos acometidos, quanto nos que recebem cuidados daqueles (LORENZ; BENATTI; SABINO, 2010; BORGES et al, 2002).

O cansaço emocional é considerado o traço inicial, podendo a manifestação ser física, psíquica ou uma combinação das duas. A despersonalização, caracterizada pela insensibilidade emocional do profissional, com prevalência de condutas cínicas e de dissimulação afetiva, é uma reação imediata após a instalação do cansaço. A baixa realização pessoal faz menção a uma auto avaliação negativa associada à insatisfação e ao desânimo com o trabalho, com sentimentos de que este não vale a pena. Portanto, em uma profissão que exige um olhar holístico e empático, a SB torna-se um grave problema de saúde publica (MOREIRA et al, 2009).

Alguns estudos apontam o crescimento do número de trabalhadores da área da saúde acometidos pelo adoecimento no trabalho, o que gera a necessidade de investimentos para identificar as causas 
desses danos e de ações que contribuam para redução dessas taxas e, como conseqüência, preserve a saúde do trabalhador.

Uma pesquisa realizada por Moreira et al (2009), com 151 profissionais de enfermagem em um hospital de grande porte da região sul do Brasil, detectou que 54 $(35,7 \%)$ dos trabalhadores apresentaram a SB. Número elevado e preocupante, pois a sintomatologia do Burnout, quando levado em consideração as áreas acometidas, pode causar grandes danos, tanto para o profissional, instituição e pacientes.

Enfermeiros, técnicos e auxiliares de enfermagem fazem parte de uma profissão caracterizada por ter, em sua essência, o cuidado e por grande parte da carga de trabalho ser o contato direto com pacientes e familiares. Entre os diferentes fatores que podem comprometer a saúde do trabalhador, o ambiente de trabalho é apontado como gerador de conflito quando o indivíduo percebe $o$ hiato existente entre $o$ compromisso com a profissão e o sistema em que estão inseridos (TRINDADE; LAUTER, 2010).

Do ponto de vista da organização do trabalho, a indefinição do papel profissional; a sobrecarga de trabalho freqüentemente justificada por falta de pessoal; a falta de autonomia e autoridade na tomada de decisões, entre outras, geram um estado de estresse crônico, identificando-se como uma das profissões de maior incidência de Burnout. A deterioração na qualidade de serviços de instituições de saúde e os altos índices de absenteísmo dos profissionais dessa área são algumas das conseqüências desse quadro, todas fortemente relacionadas com a alta taxa de incidência que caracteriza a SB (PAVLAKIS; RAFTOPOULOS; MAMAS, 2010; MOREIRA et al, 2009).

Burnout geralmente leva à deterioração do bem-estar físico e emocional. O profissional afetado pela síndrome sente-se exausto, freqüentemente está doente, sofre de insônia, úlcera, dores-de-cabeça, problemas relacionados à pressão sanguínea, tensão muscular e fadiga crônica (CARLOTTO, 2010).

No Brasil, o Decreto n ${ }^{\circ} 3.048$, de 6 de maio de 1999, aprovou o Regulamento da Previdência Social e, em seu Anexo II, trata dos Agentes Patogênicos causadores de Doenças Profissionais (Ministério da Saúde, Portaria $\left.n^{\circ} 1339 / 1999\right)$. O item XII da tabela de Transtornos Mentais e do Comportamento Relacionados com o Trabalho (Grupo V da Classificação Internacional das Doenças CID-10) cita a "Sensação de Estar Acabado" ("Síndrome de Burnout", "Síndrome do Esgotamento Profissional") como sinônimos do Burnout, que, na CID-10, recebe o código Z73.0. Portando, trata a Síndrome de uma doença e como tal capaz de provocar danos à saúde do trabalhador (TRIGO et al, 2007; 
VIEIRA et al, 2006).

São vários os fatores de risco para o desenvolvimento da SB. Para enumerá-los são levadas em consideração quatro dimensões: o indivíduo, o trabalho, a organização e a sociedade, pois todos direta ou indiretamente sofrem com os efeitos da SB. (TRIGO et al, 2007; World Health Organization, 1998).
O quadro a seguir resume as principais características referenciadas por diversos autores (Benevides-Pereira, 2002; Codo \& Menezes, 1999; Firth, 1985; GilMonte \& Peiró, 1997; Maslach, Schaufeli \& Leiter, 2001; Schaufeli \& Ezmann, 1998 apud PEREIRA, 2008) que apresentam estudos sobre a síndrome.

Quadro 1 - Resumo Esquemático dos Mediadores, Facilitadores e/ou Desencadeadores da SB

\begin{tabular}{|l|l|}
\hline \multicolumn{1}{|c|}{ CARACTERISTICAS PESSOAIS } & \multicolumn{1}{|c|}{ CARACTERISTICAS DO TRABALHO } \\
\hline Idade & Tipo de ocupação \\
Sexo & Tempo de profissão \\
Nível educacional & Tempo de instituição \\
Filhos & Trabalho por turnos ou noturno \\
Personalidade: & Sobrecarga \\
$\quad \square \quad$ Nível de resiliência, & Relacionamento entre colegas de trabalho \\
$\quad \begin{array}{l}\text { Lócus de controle, } \\
\text { Padrão de personalidade tipo A, }\end{array}$ & Assedio moral \\
$\quad \begin{array}{l}\text { Variáveis do self, } \\
\text { Estratégias de enfrentamento, }\end{array}$ & Relação profissional-cliente \\
$\quad \begin{array}{l}\text { Neuroticismo, } \\
\text { Perfeccionismo. }\end{array}$ & Tipo de cliente \\
Sentido de coerência & Ambigüidade de papel \\
Motivação & Suporte organizacional \\
Idealismo & Satisfação \\
& Nível de controle, autonomia. \\
& Responsabilidade, pressão. \\
& Possibilidade de progresso \\
& Percepção de iniqüidade \\
\hline CARACTERISTICAS ORGANIZACIONAIS & Conflito com os valores pessoais \\
\hline Ambiente físico & Falta de feedback. \\
Mudanças organizacionais & CARACTERISTICAS SOCIAIS \\
Normas institucionais & Suporte social \\
Clima & Suporte familiar \\
Burocracia & Cultura \\
Comunicação & Prestígio \\
Autonomia & \\
Recompensas & \\
Segurança & \\
\hline
\end{tabular}

Fonte: Pereira (2008) 
Para o autor acima, a maneira como essas características se combinam entre si, podem vir a postergar ou facilitar o processo da SB. Por exemplo, uma pessoa com alto nível de resiliência, em uma organização com características predisponentes ao estresse ocupacional, pode vir a resistir um maior tempo quando comparada a outro colega de trabalho. No entanto, através do tempo, ou diante do aumento dos fatores negativos na instituição, ou vindo a sofrer dificuldades em nível pessoal, este equilíbrio pode romper-se, levando-o a desenvolver a SB.

A enfermagem é a quarta profissão mais estressante do setor público. $\mathrm{O}$ excesso de atividades, dificuldades em delimitar os diferentes papeis entre enfermeiros, técnicos e auxiliares de enfermagem, falta de reconhecimento, alta carga emocional, além dos baixos salários que agravam a situação exigindo dos profissionais que tenham mais de um vínculo de trabalho resultando em uma carga mensal longa e estafante. Essa diversidade de situações sugere um quadro favorável ao desenvolvimento da SB (MUROFUSE;

ABRANCHES; NAPOLEÃO, 2005).

Do ponto de vista organizacional, a SB está altamente correlacionada com baixa moral dos trabalhadores, absenteísmo e rotatividade de pessoal, resultados organizacionais negativos e baixo nível de comprometimento com o trabalho. O baixo envolvimento e comprometimento com os resultados grupais e organizacionais afetam a qualidade no atendimento ao cliente. Uma situação preocupante visto que o profissional de enfermagem é parte integrante do atendimento ao paciente. Quando levado em consideração que é o profissional que mais tempo passa com o paciente, o quadro fica, ainda, mais agravado, pois isto afeta a oferta de atenção com qualidade (CARLOTTO, 2010).

Como forma de lidar com seus problemas físicos, os profissionais afetados, por vezes, fazem uso excessivo de tranqüilizantes, drogas e álcool. O desgaste se reflete também nas relações familiares (separações, maus tratos) e no trabalho, determinando diminuição importante do rendimento e aumento de absenteísmo (NUNES, 2008).

Vale ressaltar que apesar de a SB ser reconhecida, pelo decreto $\mathrm{n}^{\circ} 3.048$, de 6 de maio de 1999, como doença, muitas vezes os sintomas e suas conseqüências na saúde do trabalhador podem representar uma armadilha para o funcionário. Pois a própria lei trabalhista em seu art. 482, que trata da justa causa para rescisão do contrato de trabalho, possibilita que o empregado seja demitido por justa causa, por abandono de emprego, desídia ou alcoolismo no desempenho de suas funções. O que acontece é que estas causas/sintomas podem ser decorrentes da própria SB. Contudo, o decreto n³048 é de 1999 e o art. 482 é 
conteúdo do decreto lei 5452 de $1^{\circ}$ de maio de 1943, portanto espera-se que seja levado em conta o momento de efetivação de cada decreto, quando realizada uma ação trabalhista que aborde a SB (TRIGO et al, 2007; VIEIRA et al, 2006; BRASIL, 1943).

O quadro 2, a seguir, trás um resumo dos sintomas decorrentes da SB.

Quadro 2 - Resumo esquemático da Sintomatologia do Burnout

\begin{tabular}{|l|l|}
\hline \multicolumn{1}{|c|}{ FÍSICOS } & \multicolumn{1}{c|}{ COMPORTAMENTAIS } \\
\hline Fadiga constante e progressiva & Negligencia ou excesso de escrúpulos \\
\hline Distúrbios do sono & Irritabilidade \\
\hline Dores musculares e osteomusculares & Incremento da agressividade \\
\hline Cefaleias, enxaqueca & Incapacidade para relaxar \\
\hline Perturbações gastrointestinais & Dificuldade na aceitação de mudanças \\
\hline Imunodeficiência & Perda de iniciativa \\
\hline Transtornos cardiovasculares & Aumento do consumo de substâncias \\
\hline Distúrbios respiratórios & Comportamento de alto-risco \\
\hline Disfunções sexuais & Suicídio \\
\hline Alterações menstruais nas mulheres & \\
\hline & \\
\hline Falta de atenção, de concentração. & Tendência ao isolamento \\
\hline Alterações de memória & Sentimento de onipotência \\
\hline Lentidão do pensamento & Perda de interesse pelo trabalho \\
\hline Sentimento de alienação & Perda de interesse pelo lazer \\
\hline Sentimento de solidão & Ironia, cinismo \\
\hline Impaciência & Absenteísmo \\
\hline Sentimento de insuficiência & \\
\hline Baixa autoestima & \\
\hline Labilidade emocional & \\
\hline Dificuldade de auto aceitação & \\
\hline Astenia, desânimo, disforia, depressão & \\
\hline Desconfiança, paranoia & \\
\hline
\end{tabular}

Fonte: Pereira (2008)

Para o autor acima citado, as causas e os sintomas não são universais. Dependendo das características da pessoa e das circunstâncias em que esta se encontre, o grau e as manifestações são diferentes. Nem todos que estão com a síndrome apresentarão todos esses sintomas e esses podem se expressar de forma diferente em momentos diferentes na mesma pessoa.

Ainda segundo o autor, uma das formas de evitar a SB é os profissionais conhecerem os seus limites e respeitá-los. É preciso trabalhar com ética, responsabilidade, respeito pela sua equipe, bem como pelos 
usuários. Para isto se faz necessário: respeitar a carga horária, aceitando o seu limite; não se ocupar com mais de um serviço, que possa vir além da sua capacidade; deve-se permitir o lazer (dançar, ouvir música, brincar com os filhos, viver em harmonia no trabalho e em casa, ter amigos); ter um animal de estimação; para quem gosta ler um bom livro, ligar para um velho amigo, pescar, viajar, exercitar; sair da rotina trabalho-trabalho. É preciso viver, namorar, amar, permitir-se ser amado, ter fé e esperança; realizar coisas que lhe dêem prazer. O profissional deve estar em realização constante e intensa quanto a suas necessidades, e descartando coisas desnecessárias que não lhe façam bem. É preciso que o profissional se pergunte:

- O que faço e gosto de fazer?

- O que faço e não gosto de fazer?

- O que não faço e gostaria de fazer?

Reflexões como estas ajudam o profissional a priorizar coisas importantes e a descartar as que não lhe favoreçam o completo bem estar. Outro fator importante é a intervenção organizacional, pois é dever do empregador, previsto no art. 170, da constituição da República Federativa do Brasil, zelar para que haja um ambiente de trabalho sadio e respeitar o trabalhador na condição de pessoa humana (BRASIL, 1988).

Assim, debates sobre o assunto devem ser realizados entre líderes de trabalhadores, profissionais de recursos humanos, sindicatos, organizações, advogados, juízes, psicólogos, médicos e ministério público, a fim de buscar alternativas que possam dizimar ou diminuir os riscos para o desenvolvimento da SB.

\section{CONSIDERAÇÕES FINAIS}

No Brasil, a literatura encontrada nos bancos de dados utilizados não é vasta em relação ao Burnout e sua prevalência.

Desta forma espera-se que o presente trabalho seja um fomentador de mais pesquisas acerca dessa patologia, principalmente na área de enfermagem, pois os sintomas físicos, comportamentais, psíquicos e defensivos, são muito preocupantes. E ao levarmos em consideração uma área relacionada ao cuidado direto com o paciente, os danos causados podem ser fatais.

A SB em trabalhadores de enfermagem é prejudicial nas esferas individual, profissional e organizacional, porque afeta negativamente a qualidade dos cuidados de enfermagem aos pacientes, familiares e intuição em um momento em que a humanização na assistência à saúde é uma prioridade.

Conforme levantamento bibliográfico, a SB é um grave problema de saúde pública, sendo 
um grande desafio para os "atores" envolvidos, pois os profissionais de saúde estão constantemente expostos aos fatores de risco que podem desencadear a síndrome, sendo alguns deles: serviços de atenção à saúde que guardam especificidades relativas ao trato com a dor, ao sofrimento e ao malestar orgânico, emocional e social das pessoas; limitação do número de profissionais; conflitos entre membros da equipe; trabalho em turnos; elevado número de plantões assistenciais. Um ambiente extremamente estressor.

Contudo, a SB pode ser evitada, desde que a cultura da organização favoreça a execução de atividades preventivas do estresse crônico, a partir da atuação em equipes multidisciplinares, numa perspectiva de resgatar as características afetivas contidas no cotidiano de quem cuida.

\section{REFERÊNCIAS}

BORGES, Lívia Oliveira et al . A síndrome de Burnout e os valores organizacionais: um estudo comparativo em hospitais universitários. Psicol. Reflex. Crit., Porto Alegre, v. 15, n. 1, 2002. Disponível em: $<$ http://www.scielo.br/scielo.php?script=sci_ arttext\&pid=S0102$79722002000100020 \& \operatorname{lng}=$ pt\&nrm=iso $>$. Acesso em: 21 Jun 2011.

BORGES, Lívia de Oliveira; ARGOLO, João Carlos Tenório; BAKER, Maria Christina Santos. Os valores organizacionais e a Síndrome de Burnout: dois momentos em uma maternidade pública. Psicol. Reflex. Crit., Porto Alegre, v. 19, n. 1, 2006.
Disponível em:

$<$ http://www.scielo.br/scielo.php?script=sci arttext\&pid=S010279722006000100006\&lng=pt\&nrm=iso $>$. Acesso em: 21 jun 2011.

BRASIL. Decreto-lei $\mathrm{n}^{\circ}$ 5.452, de 1 de maio de 1943. Aprova a consolidação das leis do trabalho. Lex: coletânea de legislação: edição federal, São Paulo, v. 7, 1943. Disponível em: <http://www010.dataprev.gov.br/sislex/pagin as/10/1943/5452.htm>. Acesso em: 5 jul 2011.

\section{BRASIL. Constituição (1988). Constituição} da República Federativa do Brasil.

Brasília, DF: Senado Federal, 1988.

Disponível em

$<$ http://www.planalto.gov.br/ccivil_03/constit uicao/constitui\%C3\%A7ao.htm>. Acesso em: 5 jul de 2011.

CARLOTTO, Mary S.. A relação profissional-paciente e a síndrome de Burnout. Encontro - Revista de Psicologia, São Paulo, v. 12, n. 17, 2010. Disponível em: $<\mathrm{http}$ ://sare.unianhanguera.edu.br/index.php/r encp/article/viewPDFInterstitial/784/849>.

Acesso em: 5 de jul 2011.

JODAS, Denise Albieri; HADDAD, Maria do Carmo Lourenço. Síndrome de Burnout los Trabalhadores de Enfermagem de um Pronto Socorro de hospital universitário. Acta paul. enferm., São Paulo, v. 22, n. 2, 2009. Disponível em: $<$ http://www.scielo.br/scielo.php?pid=S0103$21002009000200012 \&$ script=sci_arttext $>$. Acesso em: 5 de jun 2011.

LORENZ, Vera Regina; BENATTI, Maria Cecília Cardoso; SABINO, Marcos Oliveira. Burnout e estresse entre enfermeiros terciário de um hospital universitário. Rev. LatinoAm. Enfermagem, Ribeirão Preto, v. 18, n. 6, dez. 2010. Disponível em: $<$ http://www.scielo.br/scielo.php?script=sci_ arttext\&pid=S0104$11692010000600007 \& \operatorname{lng}=\mathrm{en} \& \mathrm{nrm}=\mathrm{iso}>$. Acesso em: 26 de mai 2011. 
MOREIRA, Davi de Souza et al . Prevalência da síndrome de Burnout em trabalhadores de enfermagem de um hospital de grande porte da Região Sul do Brasil. Cad. Saúde Pública, Rio de Janeiro, v. 25, n. 7, jul. 2009. Disponível em

$<$ http://www.scielo.br/scielo.php?script=sci arttext\&pid=S0102-

$311 X 2009000700014 \& \operatorname{lng}=p t>$. Acesso em: 24 mai 2011.

MUROFUSE, Neide Tiemi; ABRANCHES, Sueli Soldati; NAPOLEÃO, Anamaria Alves. Reflexões sobre estresse e Burnout e a relação com a enfermagem. Rev. LatinoAm. Enfermagem, Ribeirão Preto, v. 13, n. 2, abr. 2005. Disponível em: $<$ http://www.scielo.br/scielo.php?script=sci_ arttext\&pid=S0104$11692005000200019 \& \operatorname{lng}=e n \& n r m=i s o>$. Acesso em: 26 mai 2011.

NUNES, Maria L.. As influências do ambiente de trabalho no surgimento da síndrome de Burnout. $2008.85 \mathrm{f}$. Monografia (Especialização em Saúde Coletiva: Saúde da Família). Universidade do Extremo Sul Catarinense, Criciúma, 2008. Disponível em:

$<$ http://www.bib.unesc.net/biblioteca/sumario /000037/00003786.pdf>. Acesso em: 5 de jun 2011.

PAVLAKIS, Andreas; RAFTOPOULOS, Vasilios; MAMAS, Theodorou. Burnout syndrome in Cypriot physiotherapists: a national survey. Health Serv Res., Chipre, v. 10, n. 63, 2010. Disponível em: $<$ http://www.ncbi.nlm.nih.gov/pmc/articles/P MC2842269/?tool=pubmed $>$. Acesso em: 26 mai 2011.

\section{PEREIRA, Ana Maria T. B.. Burnout:}

Quando o trabalho ameaça o bem estar do trabalhador. 3 ed. São Paulo: Casa do Psicólogo, 2008. Disponível em: < http://www.prt18.mpt.gov.br/eventos/2004/sa ude_mental/anais/artigos/2.pdf $>$. Acesso em: 15 de mai 2011.
SILVEIRA, Núbia de Mesquita et al . Avaliação de Burnout em uma amostra de policiais civis. Rev. psiquiatr., Porto Alegre, v. 27, n. 2, 2005. Disponível em: $<$ http://www.scielo.br/scielo.php?script=sci arttext\&pid=S0101 $81082005000200006 \& \operatorname{lng}=$ pt\&nrm $=$ iso $>$. Acesso em: 14 jun 2011.

TRINDADE, Letícia de Lima; LAUTERT, Liana. Síndrome de Burnout Entre os Trabalhadores da Estratégia de Saúde da Família. Rev. esc. enferm. USP, São Paulo, v. 44, n. 2, jun. 2010. Disponível em: $<$ http://www.scielo.br/scielo.php?script=sci arttext\&pid=S0080-62342010000200005>. Acesso em: 24 mai 2011.

TRIGO, Telma R. et al.. Síndrome de Burnout ou estafa profissional e os transtornos psiquiátricos. Rev. Psiq. Clín., São Paulo, v. 34, n. 5; p. 223-233, 2007. Disponível em: <http://www.hcnet.usp.br/ipq/revista/vol34/n 5/223.html>. Acesso em: 25 mai 2011.

VIEIRA, Isabela et al . Burnout na clínica psiquiátrica: relato de um caso. Rev. psiquiatr., Porto Alegre, v. 28, n. 3, dez. 2006. Disponível em $<$ http://www.scielo.br/scielo.php?script=sci_ arttext\&pid=S0101$81082006000300015 \& \operatorname{lng}=$ pt\&nrm=iso $>$. Acesso em: 24 mai 2011. 